\title{
ON THE SYMMETRIC ALGEBRA OF QUOTIENTS OF A C*-ALGEBRA
}

\author{
by PERE ARA $\dagger$
}

(Received 17 May, 1989; revised 3 October, 1989)

1. Introduction. Let $R$ be a semiprime ring (possibly without 1 ). The symmetric ring of quotients of $R$ is defined as the set of equivalence classes of essentially defined double centralizers $(f, g)$ on $R$; see [1], [8]. So, by definition, $f$ is a left $R$-module homomorphism from an essential ideal $I$ of $R$ into $R, g$ is a right $R$-module homomorphism from an essential ideal $J$ of $R$ into $R$, and they satisfy the balanced condition $f(x) y=x g(y)$ for $x \in I$ and $y \in J$. This ring was used by Kharchenko in his investigations on the Galois theory of semiprime rings [4] and it is also a useful tool for the study of crossed products of prime rings [7]. We denote the symmetric ring of quotients of a semiprime ring $R$ by $Q(R)$.

If $A$ is a $C^{*}$-algebra then we can consider the filter $\mathscr{F}$ of closed essential ideals of $A$, directed downwards by inclusion. We denote by $Q_{b}(A)$ the algebraic inductive limit of $(M(I))_{l \in \mathscr{F}}$, where $M(I)$ denotes the $\mathrm{C}^{*}$-algebra of multipliers of $I$, and we call it the symmetric normed algebra of quotients of $A$. Clearly $Q_{b}(A)$ is a pre-C*-algebra and its completion, i.e. the $C^{*}$-algebra inductive limit of $(M(I))_{l \in \mathscr{F}}$ is Pedersen's algebra of essential multipliers of $A$ [3], [9]. However, we shall not consider this completion here. We also note that a symmetric normed algebra of quotients has been introduced and studied recently by Mathieu [5] in the setting of ultraprime Banach algebras.

It is shown in [1] that $Q_{b}(A)$ is the bounded subring of $Q(A)$. The purpose of this note is to use some recent results of $\mathrm{N}$. C. Phillips [11] to prove a stronger relation between $Q_{b}(A)$ and $Q(A)$ (see Theorem 2.1 below). We use this theorem to obtain a characterization of the $\mathrm{C}^{*}$-algebras $A$ such that $Q_{b}(A)=Q(A)$. In particular, we see that prime $\mathrm{C}^{*}$-algebras satisfy this condition.

2. The results. Let $A$ be a $C^{*}$-algebra. We view $A$ as a subalgebra of $Q(A)$ via the regular representation $a \mapsto\left[\left(R_{a}, L_{a}\right)\right]$, where $R_{a}$ (resp. $\left.L_{a}\right)$ denotes right (resp. left) multiplication by $a$. The involution of $A$ extends to a positive definite involution on $Q(A)$ by the formula $[(f, g)]^{*}=\left[\left(g^{*}, f^{*}\right)\right]$; see $[1]$. By $[1$, Theorem 1.3$]$, we can identify $Q_{b}(A)$ with the *-subalgebra of $Q(A)$ consisting of the elements of $Q(A)$ which are bounded with respect to the partial order on $Q(A)$ obtained by taking as a positive cone the set

$$
\left\{\sum_{i=1}^{n} y_{i}^{*} y_{i} \mid y_{i} \in Q(A)\right\} \text {. }
$$

Let $R$ be a ring with unity and let $M$ be the set of elements in $Z(R)$ which are not zero-divisors in $Z(R)$. If each element in $M$ is not a zero-divisor in $R$, then we can form the central localization of $R, R M^{-1}$. The elements of $R M^{-1}$ are of the form $a b^{-1}$ where $a \in R$ and $b \in M$.

Let $A$ be a $C^{*}$-algebra and let $x$ be an element in $Z\left(Q_{b}(A)\right)$ such that $x$ is not a zero-divisor in $Z\left(Q_{b}(A)\right)$. Since $A \subset Q_{b}(A), x$ belongs to the extended centroid of $A$,

$\dagger$ This work was partially supported by CICYT grant PB 86-0353-C02-01.

Glasgow Math. J. 32 (1990) 377-379. 
$C(A)$, which is, by definition, the centre of $Q(A)$ and coincides with the centralizer of $A$ in $Q(A)$; (see [1], [6]). Now $C(A)$ is a von Neumann regular ring [6, Theorem 3.3, (2)] and, since $C(A)$ has a proper involution, it is a *-regular ring in the sense of $[2$, p. 229]. By [2, Proposition 51.3], there exists $y \in C(A)$ such that $x=x^{2} y$ and $e:=x y$ is a projection in $C(A)$. In particular $e$ is bounded and consequently $e \in Z\left(Q_{b}(A)\right)$. Since $(1-e) x=0$ we obtain $e=1$ and so $x$ is invertible in $Q(A)$. In particular $x$ is not a zero-divisor in $Q_{b}(A)$.

It follows that we can form the central localization of $Q_{b}(A)$ and it is a subalgebra of $Q(A)$.

THEOREM 2.1. If $A$ is a $C^{*}$-algebra then $Q(A)$ is the central localization of $Q_{b}(A)$.

Proof. Let $q=[(f, g)]$ be an element of $Q(A)$, where $(f, g)$ is an essentially defined double centralizer on $A$. Obviously we can assume that $f$ and $g$ are defined on the same essential ideal $I$ of $A$. Let $K_{I}$ be the Pedersen's ideal of $\bar{l}$, the norm closure of $I$ in $A$. Then $K_{I} \subset I$ and, since $K_{I}=K_{I}^{2}$, we see that $f\left(K_{I}\right) \subset K_{I}$ and $g\left(K_{l}\right) \subset K_{I}$. So $(f, g)$ induces an element of the algebra of multipliers of $K_{l}$, and clearly $K_{l}$ is an essential ideal of $A$.

Let $\left\{J_{\lambda}\right\}_{\lambda \in \Lambda}$ be a maximal family of nonzero pairwise orthogonal ideals of $A$ such that $J_{\lambda} \subset K_{I}$ and with the property that $f_{\nu_{\lambda}}$ and $g_{\nu_{\lambda}}$ are bounded. We claim that $J=\bigoplus_{\lambda \in \Lambda} J_{\lambda}$ is an essential ideal of $A$. If $J$ is not an essential ideal of $A$ then there exists a nonzero closed ideal $L$ of $A$ such that $L J_{\lambda}=0$ for all $\lambda \in \Lambda$. Now choose a nonzero element $a \in\left(L \cap K_{I}\right)_{+}$. Then by [11, Theorem 2 and Proposition 3] we obtain a unique $(T, S) \in M(\overline{A a A})$ such that $T_{\mid \overline{a A}}=f_{\mid \overline{a A}}$ and $S_{\mid \overline{A a}}=g_{\mid \overline{A a}}$. It follows that $(T, S)$ coincides with $(f, g)$ on $K, \cap \overline{A a A}$ and so the restrictions of $f$ and $g$ to $K_{I} \cap \overline{A a A}$ are bounded, which contradicts the maximality of the family $\left\{J_{\lambda}\right\}_{\lambda \in \Lambda}$.

Now set $U_{\lambda}=\left\{t \in \operatorname{Prim} A \mid J_{\lambda} \not t\right\}=\left\{t \in \operatorname{Prim} A \mid \overline{J_{\lambda}} \not t\right\}$. Then $U_{\lambda}$ are pairwise disjoint open subsets of Prim $A$, the primitive spectrum of $A$ and $U=\bigcup_{\lambda \in \Lambda} U_{\lambda}$ corresponds to $J=\bigoplus_{\lambda \in \Lambda} J_{\lambda}$. We define a function $\varphi: U \rightarrow \mathbf{C}$ by

$$
\varphi(t)=\min \left\{1, \frac{1}{\left\|f_{\left.\right|_{\lambda}}\right\|}\right\}=\min \left\{1, \frac{1}{\left\|g_{\mid s_{\lambda} \|}\right\|}\right\}
$$

if $t \in U_{\lambda}$. Then $\varphi$ is a continuous bounded function on $U$ and so by the Dauns-Hofmann Theorem [10,4.4.8] there exists $z \in Z(M(J))$ such that $z a+t=\varphi(t) a+t$ for all $a \in J$ and $t \in U$. It follows that $q_{0}:=z q$ is bounded on $J$. Since $J$ is essential in $A$ we obtain that $q_{0} \in Q_{b}(A)$. Clearly, $z$ is not a zero-divisor in $Z\left(Q_{b}(A)\right)$ and so $q=z^{-1} q_{0}$, which shows that $Q(A)$ is the central localization of $Q_{b}(A)$.

Proposition 2.2. Let $A$ be a $C^{*}$-algebra. The following conditions are equivalent:

(i) $Q(A)=Q_{b}(A)$

(ii) $Z(Q(A))=Z\left(Q_{b}(A)\right)$,

(iii) any family of pairwise disjoint open subsets of Prim $A$ is finite,

(iv) $Z(Q(A))$ is finite-dimensional,

(v) any double centralizer defined on an ideal of $A$ is bounded.

Proof. Obviously (i) $\Rightarrow$ (ii) and, by Theorem 2.1 , (ii) $\Rightarrow$ (i).

By [1], $Z(Q(A)) \cong \underset{U \in \mathscr{D}}{\lim } C(U)$ and $Z\left(Q_{b}(A)\right) \cong \underset{U \in \mathscr{D}}{\lim _{b}} C_{b}(U)$, where $\mathscr{D}$ is the family of dense open subsets of Prim $A$ and $C(U)$ (resp. $C_{b}(U)$ ) denotes the algebra of continuous 
(resp. bounded continuous) complex-valued functions on $U$. From this the implications (ii) $\Leftrightarrow$ (iii) $\Leftrightarrow$ (iv) follow easily.

It is obvious that (v) implies (i).

Assume now that (i) holds and let $(f, g)$ be a double centralizer defined on an ideal $I$ of $A$. Let $L$ be the left annihilator of $I$ in $A$. Then $L$ coincides with the right annihilator of $I, L$ is an ideal of $A$ and $I \oplus L$ is an essential ideal of $A$. By using $f(I) L=0$ and $L g(I)=0$, we see that we can extend $(f, g)$ to a double centralizer $(\tilde{f}, \tilde{g})$ on $I \oplus L$ by putting $\bar{f}(x+y)=f(x)$ and $\tilde{g}(x+y)=g(x)$ for $x \in I, y \in L$. Therefore we can assume without loss of generality that $l$ is an essential idea of $A$.

By (i) there exists an essential ideal $J$ of $A$ such that $J \subset I$ with $f_{\mid J}$ and $g_{\mid J}$ bounded. If $f$ is not bounded then there exists a sequence $\left\{x_{n}\right\} \subset I$ such that $\left\|x_{n}\right\| \leq 1$ and $\left\|f\left(x_{n}\right)\right\| \rightarrow \infty$. Now since $\bar{J}$ is essential in $A$ we have $\left\|f\left(x_{n}\right)\right\|=\left\|R_{n j \bar{j}}\right\|=\left\|L_{n \mid j}\right\|$ where $R_{n}$ (resp. $L_{n}$ ) denotes right (resp. left) multiplication by $f\left(x_{n}\right)$. It follows that there exist $z_{n} \in J$ with $\left\|z_{n}\right\| \leq 1$ such that $\left\|z_{n} f\left(x_{n}\right)\right\| \rightarrow \infty$. Since $z_{n} f\left(x_{n}\right)=f\left(z_{n} x_{n}\right)$ this leads to a contradiction. So any double centralizer defined on an ideal of $A$ is bounded and consequently $(v)$ holds.

Finally we state two immediate consequences of Proposition 2.2.

Corollary 2.3. (i) If $A$ is a prime $C^{*}$-algebra then every double centralizer defined on an ideal of $A$ is automatically continuous.

(ii) If Prim $A$ is Hausdorff then $Q(A)=Q_{b}(A)$ if and only if Prim $A$ is finite.

\section{REFERENCES}

1. P. Ara, The extended centroid of $C^{*}$-algebras, Archiv der Math. 54 (1990), 358-364.

2. S. K. Berberian, Baer ${ }^{*}$-rings, Grundlehren Math. Wiss. 195 (Springer-Verlag, 1972).

3. G. A. Elliott, Automorphisms determined by multipliers on ideals of $\mathrm{C}^{*}$-algebras, $J$. Functional Analysis 23 (1976), 1-10.

4. V. K. Kharchenko, Galois theory of semiprime rings, Algebra i Logika 16 (1977), 313-363. English trans. (1978), 208-258.

5. M. Mathieu, The symmetric algebra of quotients of an ultraprime Banach algebra, J. Austral. Math. Soc. (1990), to appear.

6. S. Montgomery, Fixed rings of finite automorphism groups of associative rings, Lecture Notes in Mathematics No. 818 (Springer-Verlag, 1980).

7. D. S. Passman, Group rings, crossed products and Galois theory, CBMS Series No. 64 (Amer. Math. Soc., 1986). 207-235.

8. D. S. Passman, Computing the symmetric ring of quotients, J. Algebra 105 (1987),

9. G. K. Pedersen, Approximating derivations on ideals of $\mathrm{C}^{*}$-algebras, Invent. Math. 45 (1978), 299-305.

10. G. K. Pedersen, $C^{*}$-algebras and their automorphism groups (Academic Press, 1979).

11. N. C. Phillips, A new approach to the multipliers of Pedersen's ideal, Proc. Amer. Math. Soc. 104 (1988), 861-867.

Departament de Matemàtiques

Universitat Autoònoma de Barcelona

08193 Bellaterra, Barcelona

SPAIN 